\title{
SCIENTIFIC REPRTS Corrigendum: Ecto-domain phosphorylation promotes functional recovery from spinal cord injury
}

Kenji Suehiro ${ }^{1}$, Yuka Nakamura ${ }^{2}$, Shuai $\mathrm{Xu}^{1}$, Youichi Uda ${ }^{1}$, Takafumi Matsumura ${ }^{1}$, Yoshiaki Yamaguchi3, Hitoshi Okamura ${ }^{3}$, Toshihide Yamashita² \& Yoshinori Takei ${ }^{4}$

Scientific Reports 4:4972; doi: 10.1038/srep04972; published online 15 May 2014; updated 01 July 2015

The original version of the Article contained errors in Affiliations 1, 3 and 4. These errors have now been corrected in the PDF and HTML versions of the Article.

${ }^{1}$ Department of Genomic Drug Discovery Science, Graduate School of Pharmaceutical Science, Kyoto University, Kyoto, Japan. ${ }^{2}$ Department of Molecular Neuroscience, Graduate School of Medicine, Osaka University, Osaka, Japan. 3Department of Systems Biology, Graduate School of Pharmaceutical Science, Kyoto University, Kyoto, Japan. ${ }^{4}$ Department of Nanobio Drug Discovery Science, Graduate School of Pharmaceutical Science, Kyoto University, Kyoto, Japan. 